\title{
Éco-conception dans le secteur des TIC et relocalisation des activités : étude de cas dans des TPE françaises
}

Ecodesign for IT Industry and Relocation of Activities : a Case Study in French $V S B$

Gilles Puel et Valérie Fautrero

\section{OpenEdition \\ Journals}

Édition électronique

URL : http://journals.openedition.org/developpementdurable/9906

DOI : 10.4000/developpementdurable.9906

ISSN : 1772-9971

Éditeur

Association DD\&T

Référence électronique

Gilles Puel et Valérie Fautrero, «Éco-conception dans le secteur des TIC et relocalisation des activités

étude de cas dans des TPE françaises », Développement durable et territoires [En ligne], Vol. 4, n

3 | Octobre 2013, mis en ligne le 30 octobre 2013, consulté le 19 avril 2019. URL : http://

journals.openedition.org/developpementdurable/9906; DOI : 10.4000/developpementdurable.9906

Ce document a été généré automatiquement le 19 avril 2019

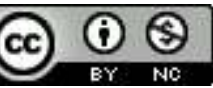

Développement Durable et Territoires est mis à disposition selon les termes de la licence Creative Commons Attribution - Pas d'Utilisation Commerciale 4.0 International. 


\title{
Éco-conception dans le secteur des TIC et relocalisation des activités : étude de cas dans des TPE françaises
}

\author{
Ecodesign for IT Industry and Relocation of Activities : a Case Study in French \\ VSB
}

Gilles Puel et Valérie Fautrero

1 La notion de "développement durable » tente aujourd'hui d'harmoniser des objectifs environnementaux et économiques, mais aussi sociaux, voire de gouvernance (Flipo et al., 2012). Si l'éco-conception a émergé dans le mouvement plus large de l'« environnementalisme industriel» (Hoffman, 1997), elle a progressivement intégré cette notion de durabilité. Elle vise à mettre sur le marché des produits ou des services en maitrisant leur impact environnemental pendant toutes les phases de leur cycle de vie. Cette prise en compte de l'impact environnemental et plus largement des objectifs de développement durable est réalisée dès l'amont du processus de production, dans la phase même de conception du produit ou du service. L'éco-conception est une « approche produit » de l'éco-responsabilité de l'entreprise, présente dans certaines stratégies de développement durable aux côtés d'autres approches plus communes telles les écoproduits - produits éco-compatibles selon les critères d'un écolabel - ou le management environnemental, qui est une approche site. Elle permet d'intégrer des préoccupations environnementales à la gestion des activités de production.

2 Bien que les premiers projets datent des années 1990, l'éco-conception reste une démarche peu courante. L'une des difficultés est liée au fait que l'éco-conception dépasse le cadre opérationnel de la conception et s'appuie sur une approche transversale touchant l'ensemble des acteurs qui interviennent dans le processus de production (conception, R\&D, fabrication) mais aussi, d'une manière élargie, les acteurs en amont et en aval de ce processus (fournisseurs, clients, distributeurs) et avec des effets variés sur l'environnement (Berkhout, Hertin, 2001). 
3 L'analyse présentée dans ce papier porte sur le secteur des technologies de l'information et de la communication limité aux activités de fabrication et de services à forte valeur ajoutée, sur la base de la définition de l'OCDE ${ }^{1}$. Or, dans le domaine des TIC, le processus de production est un processus de réseau, très souvent de réseau international distribuée spatialement : il est assez rare que la fabrication et la fin du cycle de vie du produit se réalisent dans le pays même où la conception est réalisée. Cet éclatement du processus de production comporte des implications non négligeables au niveau de la manière dont la responsabilité écologique est perçue et vécue par le producteur.

4 Eu égard à la spécificité d'un secteur TIC mondialisé et largement régulé (Huang et al., 2012), quelles peuvent être la source des motivations à l'éco-conception pour des TPE européennes? Quels peuvent être les liens entre un cadre de pensée « développement durable » mondialisé (Draetta et al., 2009) et l'émergence de pratiques d'éco-conception localisées à contre-courant des grandes logiques spatiales du secteur? L'éco-conception de produits TIC peut-elle être un moyen de relocaliser une partie de cette production en Europe? Quelles en seraient les conditions nécessaires? Quelle approche du « paradigme » du développement durable serait pertinente pour que la mise en œuvre de projets d'éco-conception dans les TIC puisse renforcer des projets de développement territorial?

5 Après avoir exposé le protocole de recherche, ce travail présente une revue de la littérature sur les freins et leviers aux pratiques de l'éco-conception dans les entreprises du secteur TIC; cette grille d'analyse sert de modèle pour esquisser des réponses à nos hypothèses et nous interroger sur les pratiques émergentes de l'éco-conception dans les TPE et les motivations des entrepreneurs.

\section{Le protocole de recherche}

La méthodologie s'appuie sur une double approche : une revue de littérature et une étude empirique à dimension comparative.

7 L'extraction des documents formant le corpus qui a servi à la revue de littérature a été opérée sur plusieurs bases de données complémentaires : Science Direct, IEEE $^{2}$ et Ebsco. Cette approche relève du choix d'ouvrir le spectre de recherche afin d'obtenir un corpus conséquent et multidisciplinaire. Comme la définition de l'éco-conception reste implicite dans la littérature, notre cadre analytique a été construit et objectivé en intégrant des synonymes ou des concepts proches tels que design for environment, ecodesign, environmentally benign design, sustainable engineering, environmentally conscious design.

Par ses différentes échelles territoriales (Monde, grande région, État, collectivités territoriales) et son approche méthodologique (desk-research internationale et enquêtes de terrain), ce projet se positionne aux côtés des études macro-analytiques sur les freins et les leviers de l'éco-conception et sur le rôle des parties prenantes (Le Pochat, 2005 ; Mathieux, 2001 ; Tukker et al., 2000) et aux côtés des études micro-analytiques sur les pratiques de l'éco-conception en entreprise (Boks, 2004; Charter, 2000; Lenox, 2000 ; McAlone, 1998, ...).

9 L'étude empirique porte sur les pratiques de l'éco-conception dans des TPE, et s'est déroulée entre 2009 et 2011. Tout d'abord, une enquête exploratoire, en ligne et par entretien, a identifié les entreprises régionales du secteur TIC des régions PACA et MidiPyrénées, pratiquant l'éco-conception, et a établi un premier tour d'horizon sur les 
représentations des freins et leviers auprès de différents acteurs concernés (industriels mais aussi institutionnels) ${ }^{3}$. Nous avons mené des entretiens exploratoires auprès de sept entreprises en Midi-Pyrénées : Ordiver (ordinateur éco-conçu, travail en réseau); Box (boîtier de communication et communication de machines); Discour (Agence de Communication, services), Asso (Assembleur d'ordinateurs, informatique durable, travail en réseau), Battery (Régénération de batteries), Encres (encre végétal), et Insert (entreprise d'insertion, reconditionnement d'ordinateurs, vente d'ordinateurs d'occasion).

Dans un deuxième temps nous avons sélectionné trois entreprises, écartant les autres soit parce qu'elles n'étaient pas enregistrées dans le secteur TIC tel que nous l'avions défini (Insert, Battery), soit pour des raisons pratiques (Encres, Box).

11 À la suite de cette sélection, plusieurs séries d'entretiens par entreprise ont été nécessaires pour interroger tous les acteurs impliqués dans l'éco-conception. Les entretiens ont été réalisés sur une période de quatre semaines pour chaque entreprise : le grand nombre de questions et le caractère discursif des entretiens prenaient beaucoup de temps et devaient s'insérer dans la temporalité d'une activité économique souvent vibrionnante ${ }^{4}$.

Tous les entretiens ont été enregistrés, puis ont fait l'objet de retranscriptions avant l'élaboration de monographies (Delanoe et al., 2011).

13 Ces entretiens semi-directifs ont suivi un protocole de recherche fondé sur nos hypothèses de travail et axé sur une grille d'analyse multicritères. Ce protocole, initialement conçu pour de grandes entreprises, en raison des nécessités de la dimension comparative de l'étude (Grands groupes ou PME en PACA et à Chongqinq) a été adapté aux réalités des PME/TPE, où les acteurs sont peu nombreux voire une personne unique.

\section{2. Éco-conception et développement durable dans les TIC : état des « lieux » des leviers et des freins}

En 1995, la constitution d'un groupe de travail sur l'éco-conception au sein du Programme pour l'Environnement des Nations Unies (PNUE) propose un élargissement de la notion d'éco-conception, englobant non seulement les aspects écologiques, mais aussi les aspects sociaux du développement des produits. L'intégration de la notion de durabilité représente un tournant dans l'histoire du concept qui, dès lors, relève du paradigme de la production et de la consommation durables (Van Weenen, 1995). L'activité d'écoconception de l'industrie des TIC prend alors son inspiration pour se développer (Stevels, 2001). Sous l'influence de nombreuses initiatives provenant à la fois des secteurs privé et public, notamment de l'U.E, force motrice sur le plan réglementaire, l'éco-conception glisse progressivement vers la « conception durable» (Linton et alii 2007).

Nous faisons l'hypothèse que si l'action écologique de l'entreprise industrielle s'inscrit dans un cadre commun situé à l'échelle globale, elle reste fondée sur une rationalité plurielle et non univoque. Le contexte socio-spatial local et/ou régional (cadre national ou supranational) est à l'origine d'interactions et de boucles de rétroactions avec l'entreprise qui influencent la décision et l'action de son responsable. C'est dans cette perspective que nous analysons la littérature qui interroge les leviers et les contraintes à l'adoption des nouvelles pratiques éco-responsables. 
Tableau 1. Les freins

\begin{tabular}{|l|l|}
\hline $\mathrm{E}$ & Pas de bénéfices environnementaux clairs \\
\cline { 2 - 3 } $\mathrm{X}$ & Désengagement des acteurs (personne ne la perçoit comme relevant de sa responsabilité) \\
$\mathrm{T}$ & \\
$\mathrm{R}$ & \\
$\mathrm{N}$ & Pas de demande des stakeholders structurée et formalisée \\
$\mathrm{E}$ & \\
$\mathrm{S}$ & Vision à court terme \\
\cline { 2 - 3 } & \\
\cline { 2 - 3 } & Pas d'obligation règlementaire \\
\hline $\mathrm{N}$ & Conflit avec les spécifications fonctionnelles \\
\cline { 2 - 3 } $\mathrm{N}$ & Manque de compétences en interne \\
\cline { 2 - 3 } $\mathrm{T}$ & Investissement perçu comme non rentable \\
\cline { 2 - 3 } $\mathrm{R}$ & Manque de temps \\
\cline { 2 - 2 } $\mathrm{N}$ & Manque de coopération entre les départements \\
\cline { 2 - 2 } $\mathrm{E}$ & Ecart trop important entre les promoteurs de l'éco-conception et ses exécutants \\
\cline { 2 - 2 } $\mathrm{S}$ & Complexités organisationnelles, le manque d'une infrastructure adéquate \\
\cline { 2 - 2 } & Nuisance commerciale \\
\hline
\end{tabular}

Source : auteurs, adapté de Delanoe et al., 2011

16 Une étude quantitative menée auprès de 150 entreprises anglaises montre que l'écoperformance d'un produit ou d'un service est positivement influencée par une série de facteurs internes et est directement proportionnelle à l'implication de l'ensemble des acteurs concernés (Pujari, 2003).

17 Pour la période 2000-2005, six études soulignent que les leviers sont présents toujours de manière combinée: il n'existe pas de moteur unique ayant conduit à l'éco-conception, mais un ensemble de facteurs dont la combinaison varie non seulement selon les entreprises concernées, mais aussi les produits visés (Lenox, 2000; Mathieux, 2001; Tukker, 2000 ; Charter, 2000). Boks (2004) valide ces mêmes résultats auprès d'entreprises asiatiques de l'électronique. 
Tableau 2. Les leviers

\begin{tabular}{|c|c|c|c|c|}
\hline \multicolumn{5}{|c|}{ Préoccupations } \\
\hline Économique & Environnementale & $\begin{array}{c}\text { Conformité } \\
\text { réglementaire }\end{array}$ & $\begin{array}{c}\text { Image de } \\
\text { l'entreprise }\end{array}$ & $\begin{array}{c}\text { Des parties } \\
\text { prenantes }\end{array}$ \\
\hline $\begin{array}{c}\text { Réduction } \\
\text { des coûts de } \\
\text { production } \\
\text { (énergie) }\end{array}$ & $\begin{array}{c}\text { Changement } \\
\text { climatique }\end{array}$ & $\begin{array}{c}\text { Directives } \\
\text { européennes }\end{array}$ & $\begin{array}{c}\text { Auto-régulation } \\
\text { du chef } \\
\text { d'entreprise } \\
\text { stakeholders : } \\
\text { clients, } \\
\text { fournisseurs, } \\
\text { financeurs, } \\
\text { groupes de pression } \\
\text { tels médias, } \\
\text { concurrents...) }\end{array}$ \\
\hline $\begin{array}{c}\text { Avantage } \\
\text { financier }\end{array}$ & $\begin{array}{c}\text { Émissions de CO } \\
\text { Léngislation }\end{array}$ & nationale & $\begin{array}{c}\text { Douligner la } \\
\text { politique } \\
\text { environnementale } \\
\text { de l'entreprise }\end{array}$ & $\begin{array}{c}\text { stakeholders } \\
\text { internes } \\
\text { (propriétaires, } \\
\text { gestionnaires, } \\
\text { employés,...) }\end{array}$ \\
\hline $\begin{array}{c}\text { Accès à de } \\
\text { nouveaux } \\
\text { marchés }\end{array}$ & $\begin{array}{c}\text { Utilisation de } \\
\text { substances } \\
\text { nocives }\end{array}$ & Normes & & $\begin{array}{c}\text { Action d'un } \\
\text { « champion }\end{array}$ \\
\hline & $\begin{array}{c}\text { Réduction de la } \\
\text { consommation } \\
\text { d'énergie }\end{array}$ & & & \\
\hline
\end{tabular}

Source : auteurs, adapté de Delanoe et al., 2011 et estime que la recherche en éco-conception a largement, voire systématiquement, négligé ces facteurs sociologiques, psychologiques, émotionnels et intangibles qui construisent ou détruisent les processus de mise en œuvre de l'éco-conception dans l'entreprise.

Les articles parus après 2006 mentionnent beaucoup moins l'éco-conception mais l'abordent par l'intermédiaire du « durable » : sur les 24 articles publiés en 2007, un tiers contiennent le mot «durable» dans leur titre et la majorité y font une référence directe dans le texte. Même les articles d'ingénierie informatique sur l'éco-conception et le reconditionnement, se situent dans le contexte de la durabilité ou du développement durable.

Cette émergence place l'éco-conception comme l'un des moyens de conception d'un produit durable (Ljungberg, 2007). Néanmoins le terme « durable » se réfère surtout à la combinaison « environnement et économie » (Flinders, 2009) et beaucoup moins souvent aux aspects sociaux et éthiques de la production. Il en est ainsi de la focalisation sur les coûts liés à la consommation d'énergie, d'autant plus forte qu'elle s'inscrit dans la conjoncture actuelle de l'augmentation du prix du pétrole et de l'électricité.

21 Néanmoins la principale limite de ces études est qu'elles portent quasiment toutes sur des grandes entreprises, quelquefois sur des PME mais rarement sur des TPE (Lepochat et al., 2007). 


\section{Résultats et discussion}

\subsection{La prégnance des piliers social et environnemental dans l'établissement d'activités éco-conçues}

D’une manière générale, les leviers analysés par la littérature sont partagés par les professionnels enquêtés que ce soit dans le champ économique, réglementaire ou de l'image de l'entreprise. Si la préoccupation de réduction des coûts n'est jamais perçue comme une motivation, cela ne signifie pas que l'engagement dans la démarche ne concourt pas à réduire ces coûts, mais plutôt que cela passe bien après les préoccupations fondamentales des chefs d'entreprises, qu'elles soient environnementales et/ou surtout sociales.

Tableau 3. Les préoccupations

\begin{tabular}{|c|c|c|c|c|c|}
\hline \multicolumn{6}{|c|}{ Préoccupations } \\
\hline sociale & environnementale & $\begin{array}{l}\text { image de } \\
\text { l'entreprise }\end{array}$ & $\begin{array}{l}\text { des parties } \\
\text { prenantes }\end{array}$ & $\begin{array}{l}\text { conformité } \\
\text { réglementaire }\end{array}$ & économique \\
\hline $\begin{array}{c}\text { Territoire } \\
\text { (tissu local, } \\
\text { France, } \\
\text { Europe) }\end{array}$ & $\begin{array}{l}\text { Engagement } \\
\text { militant }\end{array}$ & $\begin{array}{l}\text { Autorégulati } \\
\text { on du chef } \\
\text { d'entreprise }\end{array}$ & $\begin{array}{l}\text { Aides et incitations } \\
\text { publiques }\end{array}$ & $\begin{array}{l}\text { Directives } \\
\text { européennes }\end{array}$ & \\
\hline $\begin{array}{l}\text { Valeurs } \\
\text { sociales }\end{array}$ & Recyclage & $\begin{array}{c}\text { Volonté de } \\
\text { souligner la } \\
\text { politique } \\
\text { environneme } \\
\text { ntale de } \\
\text { l'entreprise } \\
\end{array}$ & $\begin{array}{l}\text { parties prenantes } \\
\text { internes } \\
\text { (propriétaires) }\end{array}$ & $\begin{array}{l}\text { Législation } \\
\text { nationale }\end{array}$ & $\begin{array}{l}\text { Avantage } \\
\text { financier }\end{array}$ \\
\hline \multirow[t]{2}{*}{$\begin{array}{l}\text { Éthique du } \\
\text { capitalisme }\end{array}$} & $\begin{array}{l}\text { Utilisation de } \\
\text { substances } \\
\text { nocives }\end{array}$ & $\begin{array}{l}\text { Recherche de } \\
\text { légitimité }\end{array}$ & $\begin{array}{c}\text { Action d'un } \\
\text { «champion } \\
\text { environnemental » }\end{array}$ & Normes & $\begin{array}{c}\text { Accès à de } \\
\text { nouveaux } \\
\text { marchés }\end{array}$ \\
\hline & $\begin{array}{l}\text { Réduction de la } \\
\text { consommation } \\
\text { d'énergie }\end{array}$ & & & Isomorphisme & \\
\hline
\end{tabular}

Source : auteurs Les tableaux 3 et 4 ont été élaborés à partir des entretiens avec des TPE de MidiPyrénées. Ainsi le volet « social » a été ajouté à la grille d'analyse issue de la littérature (tableaux 1 et

2). L'ordre de colonnes suit une hiérarchie des motivations relevées.

Les préoccupations des chefs d'entreprise interrogés recoupent celles évoquées dans la littérature, avec des différences notables. Ainsi, les dimensions sociale et environnementale prédominent. Alors que la pression des parties prenantes externes à l'entreprise est rarement citée, ce qui paraît logique si on pense au rôle généralement pionnier en éco-conception des trois entreprises étudiées, qui a contrario jouent ce rôle incitateur dans leur propre chaîne de partenaires.

Des leviers radicalement différents appartiennent au champ du social et sont fortement liés à l'engagement militant et au rôle de pionnier de ces petites entreprises. Ici l'éventail est assez large. Il mêle des préoccupations classiques d'ancrage territorial - ressources et compétences locales diminuent les coûts environnementaux et sociaux et facilitent la mise en œuvre de la démarche que ce soit pour la production ou la commercialisation - et plus innovantes voire disruptives. En effet, pour ces entrepreneurs, relocaliser une 
production est un acte éthique voire patriotique, car il engage l'entreprise et ses partenaires contre le transfert sans fin des compétences de France ou d'Europe vers l'Asie.

L'engagement dans une éthique et des valeurs sociales alternatives est lié à l'engagement personnel fort de chaque créateur. Cela se traduit par l'intégration systématique des préoccupations d'intégration dans le cycle de vie des questions de recyclage du produit, élément absent de la revue de littérature. Cet engagement militant, éthique, influe sur les relations qu'entretient l'entreprise avec ses parties prenantes et plus largement avec les territoires. Les répondants refusent d'adhérer pleinement à l'ensemble des logiques de l'économie globalisée, où les valeurs sociales sont négligées et mises à mal. Ils ont la volonté de constituer leurs propres réseaux de valeurs, dans lesquels salariés et partenaires externes à l'entreprise sont acteurs. Ainsi par effets d'entraînement, l'ensemble de la chaîne de fabrication doit être amené à évoluer et modifier ses méthodes de travail.

Tableau 4. La mise en œuvre de valeurs militantes

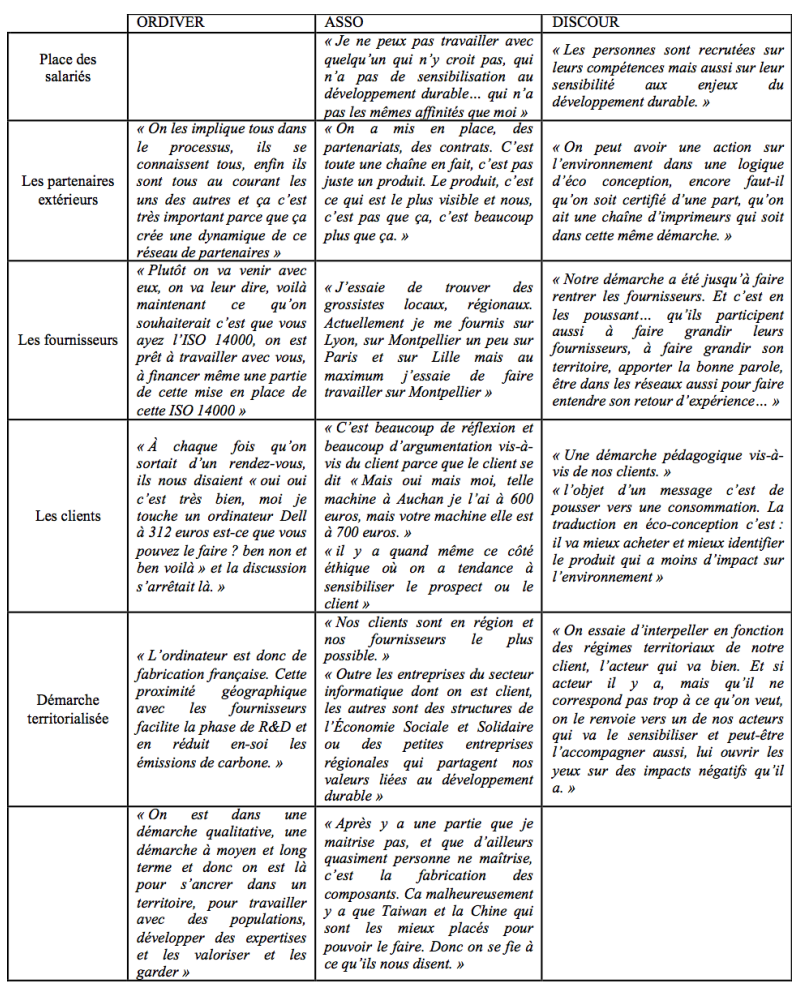

Source : auteurs, enquête 2009-2011

Le champ même de l'activité des entreprises étudiées est modifié par cet engagement, il s'élargit. La démarche de production intègre pleinement l'ensemble des partenaires pour pouvoir être cohérent (calcul du bilan carbone, par exemple). Cela modifie les activités, les rapports clients-fournisseurs et la conception du modèle économique, dans laquelle la recherche du prix le plus bas est traditionnellement le critère principal de sélection. La vision de ces entrepreneurs s'effectue à long terme, ce qui s'écarte du modèle capitaliste dominant, mais ils n'en font pas état explicitement devant leurs partenaires. "Parfois on peut nous taxer d'idéalisme, mais ceci dit c'est pas un discours qu'on sert à tout le monde non plus, parce qu'il y a des choses qu'on dit pas non plus puisqu'effectivement ça pourrait 
effrayer nos clients si je puis dire, il y a des choses qu'on tait sciemment comme par exemple la volonté de développer ce modèle. Ça peut montrer qu'on est un peu prétentieux ou donneur de leçon, ce qui faut surtout pas quand on est dans un rapport commercial avec des clients, lui montrer qu'on fait mieux les choses que lui. »

Dès lors, les freins à cette démarche sont nombreux, car l'activité élargie nécessite de fédérer les parties prenantes autour d'une communauté de valeurs, impliquant un partage de compétences et l'adhésion des partenaires, dans un contexte environnemental où la méconnaissance des uns, l'absence de repères officiels, et la volonté de manipulation des autres, créent un flou sur les notions mêmes d'éco-conception et de développement durable.

En effet, la recherche de légitimité et de conformité, participe du découplage des discours et des actions, dans un contexte où les exigences institutionnalisées tendent à contraindre de façon croissante les organisations (Rojot, 2005).

\subsection{Les limites d'une démarche socio-environnementale}

Parmi les freins relevés dans la littérature (Tableau 2), ceux centrés sur la complexité des grandes entreprises, ne concernent évidemment pas nos TPE. À l'inverse, certains freins relevés dans notre étude sont directement liés à ce type de structure, à leur fragilité, et sont renforcés par l'état de pionnier sur un produit. Beaucoup reposent sur une ou deux personnes, certes très engagées, mais qui doivent arbitrer entre le surcoût du produit et le marché, entre les caractéristiques fonctionnelles et esthétiques du produit et ses qualités environnementales, développer ou trouver les compétences qui parfois font défaut en interne, et surtout faire montre d'un trésor de patience et d'une force de conviction marketing face à des clients potentiels plus sensibles à l'économie qu'à l'environnement.

31 Trois types de verrous ressortent de notre travail et font référence i) à la faiblesse inhérente à ces petites structures, ii) à l'environnement jugé peu favorable, et enfin iii) à l'absence de marché, qui se traduit par un prix trop cher, des clients qui ne veulent pas acheter, des partenaires parfois réticents... Le tableau 5 décline ces freins. 


\begin{tabular}{|c|}
\hline le partenaires « globalisés » \\
\hline $\begin{array}{l}\text { "Notre entreprise a assez peu de poids vis-à-vis de ces fournisseurs, type Intel, où pour avoir une certaine } \\
\text { transparence sur leur mode de production c'est très compliqué, pratiquement impossible. " } \\
\text { "C'est fastidieux et ça coûte cher et ça peut se retourner contre vous actuellement. " } \\
\text { "(Les gros) ils ont des blogs des machins, tous leurs réseaux qui sont en sous-strates } \\
\text { de leur propre entité qui fonctionnent justement pour dénigrer ou mettre en avant leur } \\
\text { produit et qui fonctionnent très bien. " }\end{array}$ \\
\hline Pédagogie et accompagnement des parties prenantes \\
\hline $\begin{array}{l}\text { "Une des grosses difficultés, fédérer les différentes organisations, qui sont différentes bien sûr mais à un } \\
\text { moment donné, il faut que le monde aille vers le même point." } \\
\text { "L'aspect négatif, c'est (...) le temps passé à sensibiliser les parties prenantes, à faire avancer nos } \\
\text { fournisseurs avec nous, là aussi il s'agit pas d'avoir une offre innovante si nos fournisseurs ne peuvent pas } \\
\text { suivre, alors il faut les pousser, et ça c'est épuisant des fois." } \\
\text { " (Le client) lui expliquer qu'on produit en CAT, qu'on produit en France c'est beaucoup plus compliqué. } \\
\text { Lui il n'a pas forcément d'intérêt ou de sensibilité à ce genre de choses. Ça ne l'intéresse même pas. " }\end{array}$ \\
\hline nents et à proposer un prix « compétitif» \\
\hline $\begin{array}{l}\text { "Il y a toujours un rapport de force client/fournisseur, et donc ils vont demander tous les ans à leurs clients } \\
\text { ou fournisseurs de baisser leur prix " } \\
\text { " } \grave{A} \text { un moment, les clients, il faut qu'ils se rendent compte que le prix induit un certain mode de } \\
\text { production... au-delà de } 5 \% \text { c'est très dur à faire comprendre aux clients. Parce que le problème de nos } \\
\text { produits, c'est qu'ils rendent un certain nombre de service mais qui sont invisibles pour le client " }\end{array}$ \\
\hline Absence d'institutions normatives permettant de se distinguer des concurrents qualifiés de « malhonnêtes » \\
\hline $\begin{array}{l}\text { "Le Green Business... cela devient trop un eldorado, un peu un Far West, où il n'y a pas vraiment de } \\
\text { règles, pas vraiment de lois, où n'importe qui avec un pouvoir de communication peut vendre n'importe quoi. " } \\
\text { "Il existe beaucoup de manière de faire des bilans carbones, donc à notre sens, actuellement faire un bilan } \\
\text { carbone a peu ou pas d'intérêt. " } \\
\text { "Mais il y a aucun label en fait qui actuellement valorise la gestion du cycle, valorise la gestion sociale de } \\
\text { sa production, de son produit ou la manière dont va être utilisé le produit et tout ça. " }\end{array}$ \\
\hline
\end{tabular}

Source : auteurs, enquête 2009-2011

32 Les freins liés à l'environnement de l'entreprise sont les plus nombreux. Si nos TPE partagent l'idée que les préoccupations environnementales gagnent du terrain, elles regrettent que cela s'arrête souvent au verbe ou à un jeu d'image (marketing au mieux, greenwashing au pire). L'absence d'institutions normatives (labels, par exemple), voire coercitives, ne leur permet d'ailleurs pas de se distinguer des concurrents pratiquant le greenwashing. Et leur faible poids économique et politique vis-à-vis des partenaires "globalisés " rend incertain la qualité des ressources qu'elles leurs achètent, et les interrogent sur la transparence et la véracité des informations données par ces fournisseurs.

33 Selon elles, les partenaires ont une vision court-termiste fondée sur la quête du meilleur coût et du profit immédiat. Et dans un marché TIC globalisé, ils arbitreraient entre toutes les différences territoriales pour acheter, produire moins cher à reporter sur des législations nationales inefficaces ou plus accommodantes les questions du retraitement des déchets, profitant des « pollution haven » (Olszak, 2011). Dans les faits, les stakeholders jouent rarement le jeu ou guère longtemps; dans le meilleur des cas, leur engagement nécessite ce dont ne dispose pas les TPE : du temps pour faire de la pédagogie et emporter la conviction. En effet, l'accompagnement des parties prenantes, pour pallier leur absence de prise de conscience ou leur désintérêt des enjeux sociétaux, requiert une disponibilité et une énergie dépassant les capacités internes des petites structures. Aussi n'est-il guère étonnant que ces dernières aient le sentiment de trop jouer un rôle que devraient tenir certaines structures publiques qui se contentent souvent d'accompagner le mouvement et non de l'inciter. Si les pouvoirs publics sont systématiquement loués dans les entretiens pour avoir initié certaines actions, très rapidement l'éloge fait place à toute une série de reproches. Le premier concerne le capital risque qui est trop rare. 

l'interruption de ses activités, en fin d'année 2011. donnez le bâton pour vous faire battre ». genre de choses. Ça ne l'intéresse même pas. »

\section{Conclusion}

39 Ce papier, eu égard aux questions posées dans l'introduction, montre que, dans les pratiques de l'éco-conception des TPE du secteur TIC, les freins restent classiques (un contexte institutionnel pas vraiment favorable avec une régulation orientée vers les intérêts des grands groupes, des attentes des consommateurs émergentes mais qui butent sur la question du surcoût, un coût d'entrée très élevé, etc.). Bref, il semble ne pas exister de marché pour ce type de produits éco-conçus. visibilité, où les capital-risqueurs privés n'auraient pas voulu s'insérer ou auraient été trop gourmands pour nous... il y a aussi une concurrence vis-à-vis du crédit et des levées de fond et donc c'est de montrer que ces modèles économiques peuvent être viables et ont leur rentabilité et une rentabilité plus sûre, plus ferme, parce que justement elle est sur du long terme ».

d'une législation contraignante qu'ils souhaitent voir se renforcer. En effet, ils s'estiment en avance par rapport à la réglementation et aimeraient que celle-ci soit davantage contraignante afin d'établir (et d'institutionnaliser) leur avantage concurrentiel. Mais en même temps, ils reprochent à la législation une certaine inefficacité. Le paradoxe n'est qu'apparent: la Directive RoHS $^{5}$ a fait avancer les choses mais personne ne lutte efficacement, d'une part, contre les faux certificats, et d'autre part, contre les procédures utilisées par les grands groupes pour contourner cette réglementation. Nos répondants ont la perception d'un ensemble de règlements et d'outils de l'éco-conception inéquitable, conçu pour les grosses structures et favorisant par ailleurs un greenwashing impuni. Leur attitude à l'égard des brevets résume cet état d'esprit : «vous donnez la formulation, vous

logique d'un intéressement croissant (Akrich et al. 1988), leur statut de «pionnier " a confronté ces TPE à un contexte peu favorable à leur modèle axé sur le social, rendant improbable l'établissement d'un marché et d'un modèle d'affaires pérenne.

suite à une logique de prix très rapidement. »...." (le client) lui expliquer (...) qu'on produit en France, c'est beaucoup plus compliqué. Lui, il n'a pas forcément d'intérêt ou de sensibilité à ce

La recherche de produits à des prix tirés vers le bas et l'absence d'intérêt «écologique " et surtout social des parties prenantes ont finalement conduit une de ces TPE à

Cependant, il existe des tentatives de création dont les motivations sont originales, dans le sens où elles donnent la primeur aux pilier social et environnemental sur l'économique. Cette importance donnée au volet social par les chefs d'entreprises s'expliquent par leur généalogie : ils sont pour la plupart proches de l'économie sociale ou d'une autre vision de la société moins orientée vers le pilier économique (rejet du gaspillage, de la course au profit à tout prix,...). «L'objectif, c'est que déjà le matin je puisse me regarder dans la glace, parce que j'ai une morale, j'ai une conscience, mon associé aussi, et on a des valeurs et on est pas prêt à tout sacrifier à l'argent (ORDIVER) »... « Tout commence en 2001, lors d'un reportage TV sur les enfants qui trient les déchets sans protection dans un pays du Sud 
(ASSO) \#... « ... La vocation n'était pas de gagner de l'argent et faire de la croissance à tout prix. C'était plutôt faire bien le boulot avec des valeurs d'honnêteté, d'équité (DISCOUR)».

Cette dimension sociale se double d'une dimension territoriale «locale» voire nationale (rejet des délocalisations) et de tentatives revendiquées de construction de réseaux de partenaires territorialisés dans l'espace proche.

Les TPE étudiées sont pionnières sur un champ de l'éco-conception/développement durable en cours d'institutionnalisation, ce qui a des implications en termes de légitimité et de reconnaissance. Pas de prime au premier entrant, mais surtout des coûts...

D'autant qu'elles poursuivent un dessein éthique, engagé... dans lequel la place des piliers « environnement » et « social » prennent le pas sur le pilier « économique ». La durabilité du modèle qu'elles mettent en œuvre s'appuie sur une consommation raisonnée et la recomposition des filières de production, afin d'ancrer les activités dans les territoires, au plus proche des consommateurs.

Les représentations perçues par ces petits entrepreneurs du secteur TIC montrent un net décalage avec les discours optimistes issus de la littérature académique (qui, rappelons-le, ne concernent que des grands groupes ou PME) repris par les pouvoirs publics. Certes, cette vision organisante se fonde sur des éléments réels, notamment la pervasivité du discours environnementaliste. Tous les acteurs se sont appropriés ce discours, au moins du mieux qu'ils le peuvent. Mais ce que montre cette étude, c'est que bien souvent, cela ne reste qu'un discours, au mieux une transformation de l'image marketing de l'entreprise. Or ce verdissement, s'il semble représenter un progrès, est aussi paradoxalement un frein majeur pour les tentatives authentiques d'éco-conception. Tant que l'entreprise ne peut concilier logique de profit et engagement environnemental et/ou social, ce verrou risque de perdurer longtemps dans le cadre d'une économie libérale, et on peut rester sceptique sur la possibilité de faire levier du développement durable pour relocaliser des activités de production TIC sur les territoires des pays développés.

\section{BIBLIOGRAPHIE}

Akrich M., Callon M., Latour B., 1988, « À quoi tient le succès des innovations ? » Gérer et Comprendre, Annales des Mines, $n^{\circ} 11$ et 12 .

Berkhout, F., Hertin J., 2001, "Impacts of Information and Communication Technologies on Environmental Sustainability: speculations and evidence", Rapport OCDE, SPRU-Science and Technology Policy Research, University of Sussex.

Boks C., 2004, "The role of success factors and obstacles in design for environment: A survey among Asian electronics companies". Proceedings of the 12th IEEE international symposium on electronics and the environment (ISEE), p. 208-213. Scottsdale AZ (USA): IEEE.

Charter, M., 2000, Ecodesign on the UK electronics sector: a case study, Surrey: Center for Sustainable Design. 
Delanoe A., Draetta L., Jeanblanc C., Mereu G., Negri A. L., Puel G., 2011, « Les écosystèmes de l'écoconception dans le secteur des TIC : Écosystème de la production de connaissances, écosystème sectoriel, écosystème institutionnel, écosystème institutionnel et industriel en Chine " Projet Ecosystèmes de l'Ecoconception (ECO2) Responsable technique CDC, rapport final, 250 pages, TPT.

Draetta L., Puel G., Delanoe A., Negri A.L., 2009, The Eco-design of ICT: A Socio-technical Approach to the State of the Art, Proceedings of EcoDesign '09: 6th International Symposium on Environmentally Conscious Design and Inverse Manufacturing, Sapporo : IEEE.

Flinders K., 2009, “Green strategies put on hold for 2009”, Computer Weekly , 8.

Flipo F., Deltour F., Dobré M., Michot M., 2012, Peut-on croire aux TIC vertes ? Technologies numériques et crise environnementale, Presses des Mines, Paris, 272 p.

Hoffman A.J., 1997, From Heresy to Dogma, San Francisco, The New Lexington Press.

Huang D.Y., Liu C., Draetta L., Puel G., 2012, « La genèse de l'éco-conception en Chine dans le secteur des TIC, une exportation européenne ", Vertigo - la revue électronique en sciences de l'environnement [En ligne], Volume 12 Numéro 2 | consulté le 11 octobre 2012. URL : http:// vertigo.revues.org/12624; DOI : 10.4000/vertigo.12624

Le Pochat F. B., 2007, "Integrating ecodesign by conducting changes in SMEs", Journal of Cleaner Production, 15, p. 671-680.

Lenox M. K., 2000, “An assessment of design for environment practices in leading US Electronics firms", Interfaces, 30, 83-94.

Linton J. D., Klassen R., Jayaraman V., 2007, “Sustainable supply chains: An introduction”, Journal of Operations Management, 6, p. 1075-1082.

Ljungberg L., 2007, "Materials selection and design for development of sustainable products", Materials \& Design, 2, p. 466 - 479.

Mathieux F. R., 2001, "Ecodesign in the European Electro(on)ics Industry An analysis of the current practices based on cases studies", The Journal of Sustainable Product design, p. 233-245.

Olszak E., 2011, « Localisation des activités et développement durable des territoires : quelle interactivité ", communication au colloque Développement durable, Territoires et Localisation des entreprises : Vers une attractivité durable ? organisé par le LAREFI et l'ADERSE, Bordeaux. Rojot J., 2005, Théorie de l'organisation, 2 ème édition, Éditions Eska, 541p.

Stevels A., 2001, "Application of ecodesign Ten years of dynamic development", Proceedings of Ecodesign '01: 2nd International Symposium on Environmentally Conscious Design and Inverse Manufacturing, Tokyo, Japan, p. 905-915.

Tukker A. E., 2000, Eco-design: European State of the Art Part I: Specific studies, Brussels : EC.

Van Weenen J., 1995, “Towards sustainable product development”, Journal of Cleaner Production, 3 (1-2), p. 95-100.

\section{NOTES}

1. Cinq raisons principales justifient ce choix :

- la place de l'innovation et la convergence naturelle entre éco-conception et éco-innovation ;

- son niveau élevé de régulation ;

- sa réactivité au changement liée à sa nature innovante ; 
- le fort niveau d'application des TIC dans d'autres secteurs pour des usages de type « développement durable » générant de nouveaux impacts environnementaux.

2. Association internationale des ingénieurs en génie électrique et électronique.

3. Le taux de retour a été faible : moins de $20 \%$, ce qui, pour une enquête en ligne est néanmoins un bon résultat. Nos principales sources régionales ont été, pour Midi-Pyrénées, la CRCI (écoindustrie), l'ADEME, MPI (Midi Pyrénées Innovation - Agence de Développement Régionale), et Agate (Agence de développement départemental).

4. De plus, ces entretiens étaient couplés à de l'observation directe des pratiques en écoconception qui n'a pas été exploitée pour ce papier.

5. Restriction of the use of certain Hazardous Substances in electrical and electronic equipment, directive européenne de 2002.

\section{RÉSUMÉS}

Cet article présente les premiers résultats d'une recherche empirique sur l'émergence des pratiques de l'éco-conception dans le secteur des technologies de l'information et de la communication dans les TPE françaises. Les motivations des chefs d'entreprises donnent la primeur aux pilier social et environnemental sur l'économique. Cette dimension sociale se double d'une dimension territoriale «locale »: la durabilité du modèle mis en œuvre s'appuie sur une consommation raisonnée et la recomposition des filières de production, afin d'ancrer les activités dans leurs territoires, au plus proche des consommateurs. Cependant ces tentatives butent sur la réalité économique et l'absence de marché.

This paper presents the first results of an empirical research on the emerging practices of ecodesign in the field of information technology (IT) in french VSB. Motivations of entrepreneurs favor social and environmental pillar at the expense of the economy. This social dimension is coupled with a territorial dimension "local": sustainability of the model implemented is based on a rational consumption and the restructuring of production chains to anchor the activities in the territories, closer to consumers. However, these efforts come up against economic reality and the lack of market.

\section{INDEX}

Keywords : ecodesign, IT, sustainable development, VSB, relocation

Mots-clés : éco-conception, TIC, développement durable, TPE, relocalisation

\section{AUTEURS}

\section{GILLES PUEL}

Gilles Puel est maître de conférences HDR en Urbanisme et Aménagement, Géographe spécialisé sur les questions liant la technologie et les territoires. Ses terrains de recherche concernent les 
villes, l'urbanisation des campagnes et les grandes métropoles chinoises. Université de Toulouse, LEREPS/ETOS, 44, rue Peyrolieres 31000 Toulouse, +33 680436 607, gilles.puel@univ-tlse2.fr

\section{VALÉRIE FAUTRERO}

Valérie Fautrero est maître de conférences en Sciences de Gestion, LTCI-ECOGE, TELECOM Paristech, Institut Mines-Telecom, Université de Toulouse II, Département Sciences Économiques et Gestion, 5 Allée Antonio Machado, 31058 Toulouse Cedex 9, valerie.fautrero@telecomparistech.fr. Ses travaux portent sur le management stratégique des TIC. Elle s'intéresse aux enjeux technico organisationnels du déploiement d'infrastructures de télécommunications, aux dynamiques de marché, et aux usages des TIC. 